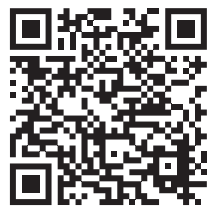

Palabras clave: Telemedicina, COVID-19.

Keywords: Telemedicine, COVID-19.

* Departamento de Consulta Externa, Instituto Nacional de Cardiología «Ignacio Chávez». Ciudad de México, México.

$\ddagger$ Sociedad Mexicana de Cardiología. Ciudad de México, México. $\S$ Departamento de Imagen Cardiovascular. Hospital Clínico San Carlos. Madrid, España.

Recibido: 22/04/2020

Aceptado:

28/04/2020

\title{
Telemedicina como instrumento de consulta cardiológica durante la pandemia COVID-19
}

\author{
Telemedicine as an instrument for cardiological \\ consultation during the COVID-19 pandemic \\ Francisco J Roldán-Gómez, ${ }^{*, \ddagger}$ Antonio Jordán-Ríos,* Amada Álvarez-Sangabriel,*,‡ \\ Carlos Guízar-Sánchez, ${ }^{*, \neq}$ Leopoldo Pérez-De Isla, ${ }^{\S}$ Luis A Lasses-Ojeda, *,‡ \\ David U Domínguez-Rivera,* Ramiro P Correa-Carrera,* Jorge E Cossío-Aranda*,‡
}

\section{RESUMEN}

La telemedicina es una herramienta subutilizada en nuestros sistemas de atención sanitaria. Se trata de un recurso tecnológico que optimiza los servicios de salud, ahorra recursos, expande la capacidad de atención especializada a lugares remotos, descongestiona servicios médicos tradicionales y es un instrumento invaluable de enseñanza e investigación. La pandemia por COVID-19 nos obliga a extender su uso y supone una oportunidad para diseñar una adecuada implementación.

\section{ABSTRACT}

Telemedicine is an underused instrument along our healthcare systems. It's a technological tool that optimizes resources, save money, expands our capacities, decongests our traditional medical services and is an invaluable help for teaching and research. The COVID-19 pandemic is forcing us to expand its use and it gives us the opportunity to design an appropriate implementation.

\section{INTRODUCCIÓN}

$\mathrm{E}^{\mathrm{i}}$ I día 11 de marzo de 2020, la Organización Mundial de la Salud (OMS) declaró como pandemia la enfermedad por COVID-19 y una de las medidas para mitigar su impacto sobre nuestros limitados sistemas de salud ha sido el confinamiento de la población en sus hogares. En este contexto, resulta lógico que se haya incrementado en forma exponencial el uso de herramientas muy básicas de telemedicina (Tm), especialmente llamadas convencionales, videollamadas y aplicaciones de mensajería. En muchos casos esto ha ocurrido sin una planeación adecuada, sin lineamientos definidos y sin herramientas que permitan la evaluación de resultados ni establecer estándares de calidad.

La Tm se encuentra íntimamente ligada al desarrollo de las telecomunicaciones, siendo tan antigua como el mismo telégrafo.
Sus mayores avances han venido de la mano de conflictos bélicos, catástrofes naturales, progresos tecnológicos, especialmente en la industria aeroespacial, y durante la expansión de enfermedades infectocontagiosas, como es el caso que nos ocupa. Debe ser considerada como un recurso tecnológico que optimiza los servicios de salud, ahorra recursos, expande la capacidad de atención especializada a lugares remotos y descongestiona servicios médicos tradicionales. Adicionalmente, es una herramienta invaluable de enseñanza e investigación.

La Tm no es sólo un concepto científico y técnico, sino que involucra aspectos administrativos, éticos, legales, económicos, deontológicos, políticos e incluso filosóficos. Esperamos que este artículo ayude a contextualizarla y a visualizar el área de inmensa oportunidad que representa dentro del ejercicio de la cardiología y de la reorganización necesaria de nuestros servicios médicos. 


\section{LA TELEMEDICINA COMO PARTE DE UNA ATENCIÓN CARDIOLÓGICA INTEGRAL}

La Tm debe entenderse como un complemento que debe estar regulado e integrado dentro de los propios procesos de la atención médica. Requiere de criterios claros de funcionamiento, protocolos que consideren los aspectos propios de cada individuo, de cada patología y de cada contexto organizativo, incluso de una reorganización profunda de los sistemas de salud que permita optimizar sus capacidades.

El éxito de la Tm, entendido éste como el logro de una mejor atención médica y de sistemas de salud más eficientes, dependerá de que su implementación esté precedida de un cuidadoso diagnóstico situacional, cuente con una planeación bien diseñada e incluya en su funcionamiento sistemas de evaluación y monitoreo. Idealmente, debe contar con marcos regulatorios modernos, aspectos de seguridad y de confidencialidad muy claros, herramientas de telediagnóstico y de telemonitoreo confiables y validadas, capacidad para el respaldo digital de documentos, así como de herramientas docentes y de investigación. Una vez integrada de esta manera, no se concebirá el mundo de la cardiología sin el concurso de la Tm y de todas sus capacidades.

\section{CONCEPTO, EXPERIENCIA E INDICADORES EN EL INSTITUTO NACIONAL DE CARDIOLOGÍA DE MÉXICO}

En la fase 2, o de dispersión comunitaria, de la pandemia por COVID-19, existe mayor riesgo de contagio al otorgar una consulta presencial tanto para el paciente como para familiares y/o personal sanitario, y existe una mayor demanda de camas y ventiladores. Por lo cual se vuelve prioritario organizar procesos (los cuales se resumen en la Figura 1).

En la fase 1, llamada de contingencia, todas las consultas presenciales y cirugías electivas se suspenden por el alto riesgo de contagio. Se difunden medidas preventivas y es, desde esta fase, cuando el uso de la Tm en la consulta cardiológica cobra vital importancia, ya que permite:
- Contener diseminación comunitaria del virus SARS-CoV-2, protegiendo a los pacientes con enfermedad cardiovascular que tienen mayor riesgo de adquirirla $y$, al mismo tiempo, al personal de salud.

- Prevenir las descompensaciones de la enfermedad cardiovascular (ECV); evitando la saturación del servicio de urgencias.

La OMS ha definido a la Tm como uso de redes de comunicación multimedia para otorgar una consulta médica cuando la distancia es un factor determinante. En esta plataforma se puede utilizar voz y datos con formato analógico y/o digital. Puede otorgarse en tiempo real mediante teleconferencia, que requiere infraestructura de banda ancha, o en tiempo diferido o asíncrona, de forma no urgente, con almacenamiento de la información que se puede revisar tiempo después.

En la cardiología y en países de ingresos bajos su uso puede ser trascendente, incluso con menor coste que lo presencial. Se puede otorgar para reducir la sobrecarga de consulta y en época de pandemia, para evitar contagios en salas de espera; en el paciente con emergencia, como es un infarto agudo del miocardio, la Tm puede asegurar no sólo el traslado y referencia eficiente del paciente a un hospital con disponibilidad de sala de hemodinámica para angioplastia coronaria, sino también para asegurar un tratamiento adecuado en el momento agudo y antes de llegar al hospital. Los pacientes en zonas rurales también pueden beneficiarse a pesar de las limitaciones en recursos, ya que permite el acceso a servicios de salud con alto nivel de especialización y optimizar recursos. Se puede implementar en hospitales no tan especializados, en donde el personal es adiestrado mediante $\operatorname{Tm}$ y, además de mejorar la calidad de salud, pueden establecerse modelos de evaluación y cooperación en investigación.

El Instituto Nacional de Cardiología «lgnacio Chávez» otorga más de 120 mil consultas al año a personas de las poblaciones más desfavorecida de México, las cuales no tienen acceso a servicios de seguridad social. Durante esta época de pandemia se ha otorgado la consulta mediante teléfono en las 14 clínicas de atención cardiovascular especializada a estos pacientes, aplicando una encuesta COVID-19 para detectar casos 
sospechosos y, también, identificando los casos de alto riesgo y grupos vulnerables. Asimismo, la información se ha difundido con el uso de redes sociales como Facebook, Instagram y Twitter. Además, se establecieron grupos de trabajo para optimizar la estancia en hospital y en casa.

Los médicos de cada clínica hacen una evaluación de los expedientes, anticipándose una semana a su cita programada, detectan mediante un triaje a los pacientes de más alto riesgo, clasifican a pacientes con riesgo bajo (verde), moderado (amarillo) y rojo (alto). Además, identifican a pacientes que ya no requieren la atención presencial en el INCICh (violeta) y que posteriormente pueden ser referidos mediante «resumen clínico-referencia contra-referencia» a otros hospitales.

En el expediente electrónico y físico se coloca una nota con la leyenda «Orientación telefónica durante la pandemia COVID-19»,
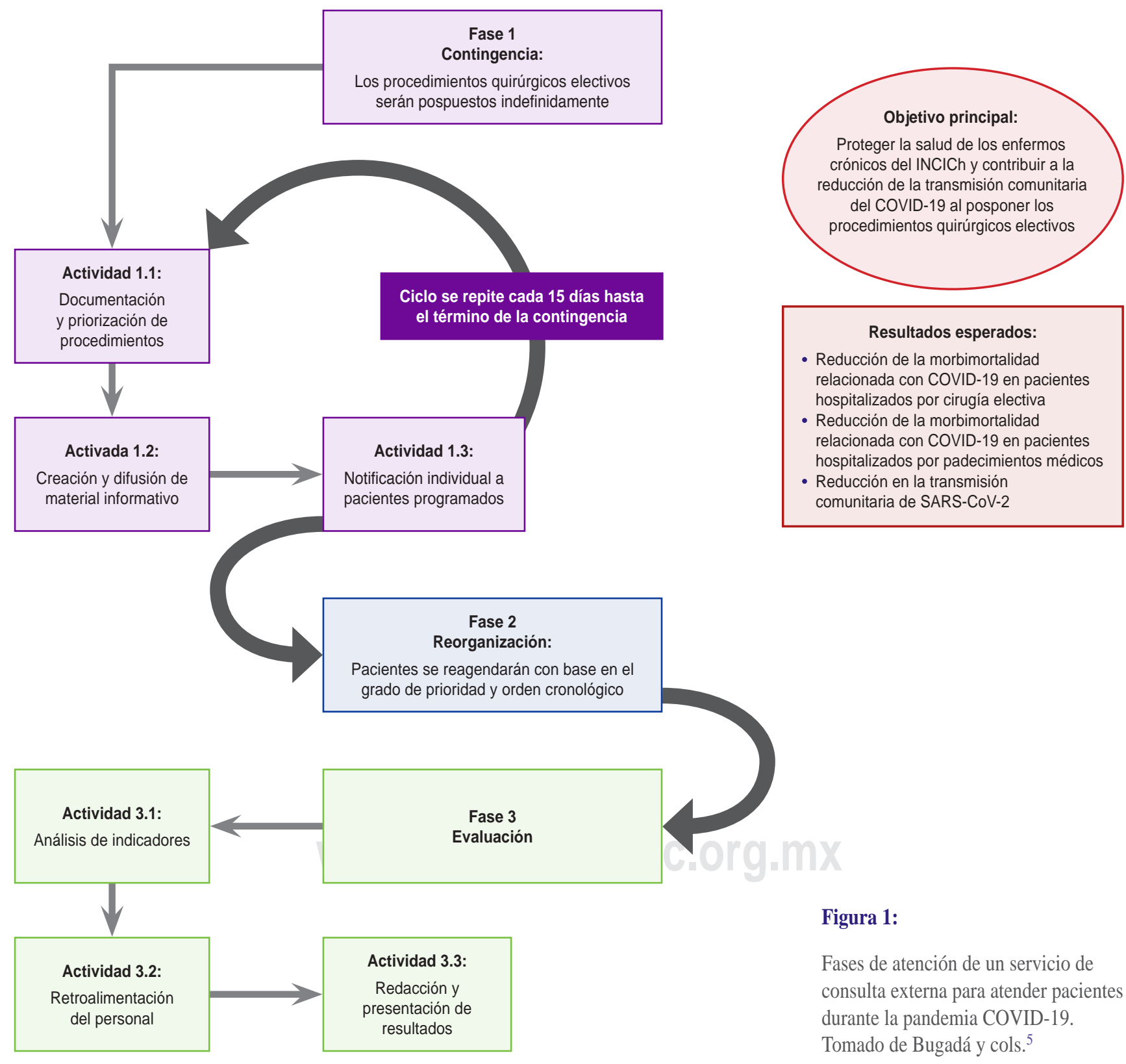
siguiendo los lineamientos de la Norma Oficial Mexicana para expediente clínico y del Comité de Expediente en nuestra institución. Se hace especial mención a su estado de salud actual, a síntomas y signos de COVID-19 y a la información sobre la pandemia y prevención por el médico que hace la consulta telefónica.

De igual modo, se hacen consensos en los grupos médicos, tomando en consideración la evidencia científica y posicionamiento de las sociedades más importantes del mundo y de nuestro país sobre el uso de medicamentos (v.g. IECA, inhibidores de receptor AT), así como el manejo médico en general y, en particular, lo cardiovascular de los pacientes con COVID-19, esto con la finalidad de crear información veraz, con ética y privacidad hacia nuestros enfermos. Sin embargo, esta información puede compartirse a través de Tm no sólo con el personal activo, sino con otras instituciones.

\section{ATENCIÓN EN PERSONAS MAYORES CON PATOLOGÍA CARDIOVASCULAR EN TIEMPOS DE COVID-19}

En los últimos años existe un incremento vertiginoso de la población de personas mayores y la principal causa de morbimortalidad en este grupo etario es la patología cardiovascular. ${ }^{1} \mathrm{La}$ información proporcionada por la Organización Mundial de la Salud y la evidencia científica actual generada a nivel mundial indican que las personas mayores son más vulnerables a presentar complicaciones y elevadas tasas de mortalidad. ${ }^{2}$

El Servicio de Cardiología Geriátrica del Instituto Nacional de Cardiología «lgnacio Chávez» ha implementado desde hace varios años un sistema de clasificación y atención vía remota (telefónica y correo electrónico); medidas con las que se ha conseguido una reducción de alrededor de $80 \%$ de visitas al Servicio de Urgencias por parte de nuestros pacientes. Dentro de la valoración global cardiogeriátrica que se realiza por Tm se encuentran los siguientes puntos:

- Anamnesis general con énfasis en aspectos cardiovasculares.

- Conciliación de medicamentos y revisión de fármacos potencialmente inapropia- dos de acuerdo a los criterios de Beers y STOPP/START.

- Funcionamiento: se valora la capacidad funcional para realizar las actividades básicas de la vida diaria por medio del índice de Katz y las actividades instrumentadas de la vida diaria a través de la escala Lawton y Brody. Por otra parte, se valora el síndrome de fragilidad por medio de la escala de FRAIL y el riesgo de desarrollar úlceras de presión a través de la escala de Norton, así como identificación de síndrome de caídas e indagatoria sobre su mecanismo y consecuencias.

- Cognitivo: escrutinio sobre datos que sugieran delirium en la última semana, utilizando el método de evaluación del síndrome confusional agudo (CAM).

- Nutricional: valoración del riesgo nutricional por medio de la escala Mini-Evaluación Nutricional Formato Corto (MNA SF) y la identificación de la presencia de probable sarcopenia en la persona mayor (SARC F).

- Afectivo: evaluación del estado afectivo de la persona mayor a través de la escala geriátrica de depresión de 15 ítems (GDS-15). ${ }^{3}$

La valoración por Tm nos permite identificar a pacientes de alto riesgo, como lo son: insuficiencia cardiaca descompensada en el último mes, síndrome coronario crónico con incremento considerable de los síntomas en el último mes, hipertensión arterial de difícil control, ${ }^{4}$ deterioro del estado funcional previo en el último mes, antecedente de haber presentado delirium en la última semana, síndrome de caídas de reciente aparición (última semana), trastorno depresivo mayor o trastorno de ansiedad con síntomas severos que atenten contra la integridad del paciente o su familia/cuidador en el último mes. De esta manera se prioriza la atención presencial de estos últimos para realizar una intervención oportuna y atenuar complicaciones.

Consideramos que esta forma de evaluación es una alternativa útil en esta pandemia y debe considerarse como un método permanente tanto para futuras contingencias como para no saturar los servicios de salud en unidades donde se brinde atención a pacientes vulnerables, especialmente a los adultos mayores. 


\section{RELACIÓN MÉDICO-PACIENTE EN Tm}

La Tm tiene dos vertientes: la primera es la relación que se establece entre profesionales o instituciones sanitarias, y la segunda es la relación entre un profesional o institución sanitaria y el paciente. Este segundo aspecto lo abordaremos en este apartado de una forma esquemática.

La demanda de servicios médicos es creciente, pero se encuentra con limitaciones, ya sea porque los pacientes, debido a su envejecimiento y a otras circunstancias, presentan una movilidad reducida, a que los pacientes y los profesionales de la salud están geográficamente muy alejados unos de otros, o a situaciones (como la pandemia actual) que hacen que sea preferible evitar los contactos personales. ${ }^{5}$ En estas situaciones la Tm se sitúa como una herramienta eficaz y segura. Su costo puede, en ocasiones, ser mayor que el de una consulta tradicional, pero la popularización de las nuevas tecnologías hace que cada vez los costes sean menores. Hay que recordar este punto: la Tm no solamente es realizar una videoconferencia con un sistema de alta calidad, sino que la comunicación mediante sistemas seguros de mensajería, videoconferencias con sistemas sencillos o simplemente la llamada telefónica, también pueden y deben ser considerados como formas de Tm.

La Tm hace que una serie de aspectos en la relación médico-paciente tome una especial relevancia. ${ }^{6}$ En primer lugar, existe una serie de aspectos técnicos relacionados con las tecnologías de los medios de comunicación utilizados. Para ello se requiere una mínima capacitación por parte del médico y del paciente. Por otra parte, los aspectos de la relación interpersonal son modificados por el tipo de elemento de comunicación, el tipo de paciente, el tipo de agente sanitario y el tipo de relación que se establece entre ambos. Uno de los principales problemas que se presenta con el uso de la Tm en la relación médico-paciente es la pérdida de elementos que todos los profesionales sanitarios usamos a diario y, muy especialmente, uno de ellos es la empatía, es decir, la confianza que se crea entre el paciente y su médico. Para un primer contacto entre médico y paciente, esta empatía puede estar muy ausente; aunque lo cierto es que cuando la Tm se usa después de una primera consulta tradicional en la que el médico y el paciente ya se han conocido, el paciente suele estar mucho más receptivo al empleo de las tecnologías. Existen, en ocasiones, elementos como personal técnico, que asiste durante la teleconsulta para facilitar el uso de las tecnologías, que pueden alterar esa empatía.

También se debe tener en cuenta que cuando la relación médico-paciente se establece mediante $\mathrm{Tm}$, se priva a la relación de dos aspectos como son el tacto y la forma no verbal de comportarse. Sin embargo, no todo son desventajas en la relación médico-paciente, ya que la Tm puede potenciar la participación del paciente a narrar sus vivencias más personales al no estar físicamente delante de una persona. Por otra parte, el aspecto novedoso que aporta al paciente la tecnología empleada para tal fin puede constituir un elemento atractivo. ${ }^{7}$

Para evitar situaciones inapropiadas se deben desarrollar normas que rijan el tipo de contactos médico-paciente en Tm. Existen pocas guías de actuación que definan cómo debe ser, pero deberían establecerse una serie de normas que aborden los problemas que pueden surgir, incluyendo las correspondientes normas éticas y normas de conducta. Los aspectos éticos que deben regir la teleconsulta deben ser los habituales de cualquier relación médico-paciente, destacando la confidencialidad como pieza fundamental. Las reglas legales en este tipo de relación son complicadas de establecer, ya que cada país tiene su propia legislación y es difícil establecer normas para una técnica que cambia día a día con una velocidad vertiginosa. Cada situación debe ser tratada de forma individualizada y contrastada con el código legislativo adecuado en cada lugar y en cada momento.

Por tanto, para concluir, podemos decir que la Tm es un tipo de relación médico-paciente con un crecimiento rápido y constante y que, pese a que presenta una serie de limitaciones y desventajas, cuando la comparamos con la relación médico-paciente tradicional presenta otra serie de ventajas. Dependiendo del tipo de Tm que se use, de los problemas médicos que se trate y de las tecnologías seleccionadas se deben crear una serie de reglas para conseguir asegurar la eficacia de esta nueva aproximación al manejo de los pacientes. 


\section{LA TELEEXPLORACIÓN Y FUTUROS PREVISIBLES}

Si bien el examen físico del enfermo genera una estrecha relación médico-paciente, la teleexploración en caso necesario, como es la emergencia de una pandemia, se convierte en una gran herramienta. ${ }^{8}$ ¿Qué tanto es el futuro de muchas de las consultas que se darán en el ámbito de la cardiología después de la pandemia COVID-19? La cardiología tiene la ventaja que además de que los signos vitales delatan a la víscera cardiaca, el corazón emite ruidos que pueden ser interpretados como fisiológicos y/o patológicos y pueden transmitirse; asimismo los estudios como electrocardiograma y ecocardiograma pueden también interpretarse hoy en tiempo real. Otros métodos que son de telemetría y Tm son el monitoreo ambulatorio de presión arterial y electrocardiograma mediante sistema Holter y de dispositivos móviles. Además, los monitoreos pueden ser de corto y largo plazo, cuya información es inmediata, sobre todo en pacientes con alto riesgo de tener arritmias malignas.

A través de la telecardiología puede haber enlace directo entre el médico de primer contacto y el cardiólogo para resolver algún problema urgente del paciente. Entre las herramientas posibles de teleexploración se encuentran:

1. Estetoscopio digital y fonocardiograma. Esto permite una auscultación digital hacia un software en la terminal del médico cardiólogo. Se requiere de un microprocesador y amplificador de la señal para su análisis espectral.

2. Registro electrocardiográfico. Puede ayudar en prácticamente todos los pacientes con enfermedad cardiovascular, pero también en situaciones urgentes como un infarto del miocardio y/o arritmias.

3. Imagen digitalizada.

\section{CONCLUSIÓN}

La telecardiología es la aplicación que ayuda a prevenir, diagnosticar y dar tratamiento a pacientes con enfermedad cardiovascular, y que además en etapa de pandemia también evita la diseminación del virus entre enfermos, familiares y personal sanitario; asimismo, ayuda a evitar la saturación de los servicios de urgencia.

\section{BIBLIOGRAFÍA}

1. Institute for Health Metrics and Evaluation. Disponible en: http://www.healthdata.org/mexico?language $=149$ [accesado el 15 de abril del 2020].

2. Liu K, Chen Y, Lin R, Han K. Clinical features of COVID-19 in elderly patients: A comparison with young and middle-aged patients. J Infect. 2020; 80 (6): e14e18.

3. Merrell RC. Geriatric Telemedicine: background and evidence for telemedicine as a way to address the challenges of geriatrics. Healthc Inform Res. 2015; 21 (4): 223-229.

4. Schwamm L, Chumbler N, Brown E, Fonarow G, Berube D, Nystrom K et al. Recommendations for the implementation of telehealth in cardiovascular and stroke care: a policy statement from the American Heart Association. Circulation. 2017; 135 (7): e24e44.

5. Gras G. Use of telemedicine in the management of infectious diseases. Med Mal Infect. 2018; 48 (4): 231-237.

6. Wootton R, Darkins A. Telemedicine and the doctorpatient relationship. J R Coll Physicians Lond. 1997; 31 (6): 598-599.

7. Luz PLD. Telemedicine and the doctor/patient relationship. Arq Bras Cardiol. 2019; 113 (1): 100-102.

8. Araiza-Garaygordobil D, Illescas-González E, CossíoAranda J, Kuri-Alfaro J, Guadalajara Boo F. El valor clínico del examen físico en cardiología: revisión de la evidencia. Arch Cardiol Méx. 2017; 87 (4): 265-269.

\section{Correspondencia:}

Jorge E Cossío-Aranda

E-mail: doctorjorgecossio@yahoo.es

\section{RESPONSABILIDADES ÉTICAS}

Protección de personas y animales. Los autores declaran que para esta investigación no se han realizado experimentos en seres humanos ni en animales.

Confidencialidad de los datos. Los autores declaran que han seguido los protocolos de su centro de trabajo sobre la publicación de datos de pacientes. 